\title{
Estudio de validación de un cuestionario de apego en adolescentes
}

\author{
JAVIER MORÁN K. ${ }^{1}$, FELIPE LECANNELIER A. ${ }^{2}$, JORGE RODRÍGUEZ T. ${ }^{3}$ \\ 1. Escuela de Psicología, Universidad de Valparaíso. \\ 2. Centro para el Estudio del Apego y la Regulación (CARE), Universidad del Desarrollo. \\ 3. Escuela de Salud Pública, Facultad de Medicina, Universidad de Chile.
}

\begin{abstract}
Validation study of attachment questionnaire in adolescents

Introduction: There are not enough specially developed tools to study attachment during adolescence in our country. The objective of this research is to adapt and validate the AAQ (Adolescent Attachment Questionnaire) for the Chilean population. Patients and Method: A cross-sectional study was conducted in 199 adolescents between 11 and 19 years old. The AAQ is a self-report questionnaire consisting of 9 items, three scales of three statements each: a) Availability scale; b) Goal corrected Partnership scale and c) Angry distress scale. The items were answered with Likert-type responses. The AAQ was originally validated in a sample of 824 adolescents, 691 of which corresponded to non-clinical population and 133 corresponded to clinical population, demonstrating satisfactory internal reliability. To create the Spanish version of the questionnaire, a translation-retranslation procedure was used evaluating semantic, content, construct and reliability validity. Results: The results showed adequate internal consistency of the instrument with Cronbach alpha's values between 0.52 and 0.74 . Also, the factorial structure of the AAQ English version was replicated. Finally, temporary rules for the interpretation of results that consider age differences for one of the 3 scales are presented. Conclusions: It is concluded that the instrument is suitable for use because it is a short self-administered questionnaire that shows similar psychometric characteristics to the original English version.
\end{abstract}

(Key words: Validation, attachment, adolescence, questionnaire).

Rev Chil Pediatr 2014; 85 (4): 437-442

\section{RESUMEN}

Introducción: Existe escasez de instrumentos especialmente desarrollados para estudiar el apego durante la adolescencia en nuestro país. El objetivo de esta investigación fue adaptar y validar a la población chilena el AAQ (Adolescent Attachment Questionnaire). Pacientes y Método: Estudio de tipo transversal, efectuado en 199 adolescentes de entre 11 y 19 años. El AAQ como cuestionario de autorreporte consistió en 9 ítems, distribuidos en 3 escalas de forma equitativa: a) disponibilidad; b) cooperación en la relación y c) señales de

Recibido el 19 de marzo de 2013, última versión aceptada para publicación el 21 de abril de 2014.

Potenciales conflictos de interés: Este trabajo cumple con los requisitos sobre consentimiento /asentimiento informado, comité de ética, financiamiento, estudios animales y sobre la ausencia de conflictos de intereses según corresponda. 
enojo y angustia. Los ítems se respondieron a través de la escala Lickert. El AAQ originalmente fue validado en una muestra compuesta por 824 adolescentes, de los cuales 691 correspondía a población no clínica y 133 correspondían a población clínica, demostrando satisfactoria confiabilidad interna. Para la elaboración de la versión en español del cuestionario se utilizó el procedimiento de traducción-retraducción, evaluándose posteriormente la validez semántica, de contenido, de constructo y confiabilidad del mismo. Resultados: Los resultados muestran una adecuada consistencia interna del instrumento con valores de alfa de Cronbach entre 0,52 y 0,74 . Además se replica la estructura factorial de la versión en inglés del AAQ. Finalmente, se entregan normas provisorias para la interpretación de los resultados que consideran diferencias de edad para una de las 3 escalas. Conclusiones: Se concluye que el instrumento resulta adecuado para su uso, en cuanto es un cuestionario autoaplicado que resulta muy breve y demuestra características psicométricas similares a la versión original en inglés.

(Palabras clave: Validación, apego, adolescencia, cuestionario).

Rev Chil Pediatr 2014; 85 (4): 437-442

\section{Introducción}

La teoría del apego, desarrollada por John Bowlby $^{1}$, inicialmente permitió describir un sistema biológicamente organizado de conductas orientadas al mantenimiento de la seguridad en infantes a partir de la cercanía con una figura (figura de apego), que tempranamente otorga cuidados. Sin embargo, la investigación posterior con adolescentes y adultos ${ }^{2-3}$ ha permitido afirmar que las relaciones de apego posteriores a la niñez también cumplen un rol importante en el ajuste general del individuo ${ }^{4}$.

Comparativamente, la mayor parte de la investigación en apego y psicopatología se ha focalizado largamente en niños, y sólo recientemente en adolescentes ${ }^{5}$, pese a que sabemos en la actualidad que factores como la disponibilidad parental y el soporte emocional son experiencias que, consistentemente permiten dar cuenta del desarrollo, inicio y mantención de psicopatología en esta edad ${ }^{4}$.

En este sentido, la comprensión de que el apego resulta una herramienta fundamental para describir y explicar las relaciones entre padres y adolescentes, ha llevado al desarrollo de una serie de instrumentos que permiten medir y/o evaluar la cualidad de este sistema.

Pese al desarrollo reciente de diversos instrumentos para evaluar el apego en adolescentes, es necesario destacar que "la mayor parte de éstos han sido desarrollados por autores que han liderado la investigación de apego principalmente en Estados Unidos y Europa", así también, es importante considerar que no to- dos han sido validados en nuestro país. Martínez y Santelices ${ }^{7}$ presentan una exhaustiva revisión de la evaluación del apego y concluyen que en Chile no existe una tradición en la investigación del apego en adultos, insistiendo además en la escasez de literatura nacional en esta temática.

El instrumento de referencia en la evaluación de la organización del apego en adolescentes ha sido la entrevista de Apego Adulto (Adult Attachment Interview) $\mathrm{AAI}^{8}$. Pese a los aportes que ha otorgado este instrumento a la investigación destacan importantes dificultades relacionadas a la cantidad de tiempo, la complejidad para su aplicación e interpretación y especialmente el hecho de que no fue desarrollada para evaluar el apego de forma específica en adolescentes. Por lo tanto, la falta de instrumentos específicos y validados para la evaluación del apego en la adolescencia, ha limitado el desarrollo de la investigación en esta área.

El objetivo de esta investigación fue adaptar y validar a la población chilena el Cuestionario de Apego Adolescente "The Adolescent Attachment Questionnaire (AAQ)". Su fácil y rápida aplicación y su bajo costo, hacen altamente viable la investigación a través de éste y abre importantes posibilidades de investigación en torno al apego en la adolescencia. Es importante señalar que la validación y adaptación del AAQ a Chile resulta pionera en cuanto al uso de cuestionarios con estas características para la investigación del apego en la adolescencia y el abordaje en este campo. 


\section{Pacientes y Método}

\section{Participantes}

Para el desarrollo de este estudio se trabajó con dos grupos. El primero estuvo compuesto por 30 adolescentes, hombres y mujeres entre 13 y 18 años, de un colegio particular subvencionado de la comuna de Valparaíso con quienes se evaluó la validez semántica del instrumento traducido al español. El segundo grupo, con el que se evaluaron las características psicométricas del instrumento, estuvo compuesto por 199 adolescentes (101 hombres y $98 \mathrm{mu}$ jeres) entre 11 y 19 años $(14,36 \pm 2,594$ años $)$, estratificándose la muestra en tres grupos etarios (11-13, 14-16 y 17-19) según adolescencia temprana, media y tardía, tal como se señal en la tabla 1.

Los participantes pertenecieron a 2 colegios particulares subvencionados, un colegio particular y primer año de psicología de una Universidad de la V Región.

Se contó con la autorización de los colegios y el consentimiento de cada una de las personas, quienes participaron voluntariamente.

\section{Instrumento}

The Adolescent Attachment Questionnaire (AAQ) fue publicado el año 1998 por West, Rose, Spreng, Sheldon-Keller y Adam ${ }^{4}$ en el Journal of Youth and Adolescence. El instrumento fue elaborado a partir de los planteamientos de Ainsworth ${ }^{9}$ y Bowlby ${ }^{1}$ en relación al apego, derivándose teóricamente 3 escalas. Este instrumento está diseñado para evaluar la percepción sobre la disponibilidad y responsividad de la figura de apego del adolescente, y no entrega un índice de seguridad o inseguridad en la relación.

El AAQ es un cuestionario de autorreporte que consta de 9 ítems, distribuidos en las siguientes 3 escalas de forma equitativa: a) disponibilidad; b) cooperación en la relación y c) señales de enojo y angustia. Los ítems se responden a través de una escala Lickert, con valores que van desde completamente de acuerdo $^{1}$ a completamente en desacuerdo ${ }^{5}$.

El AAQ originalmente fue validado en una muestra compuesta por 824 adolescentes, de los cuales 691 correspondía a población no clí-
Tabla 1. Distribución según etapa de la adolescencia

\begin{tabular}{|lcc|}
\hline Válidos & n & \% \\
\hline Adolescencia temprana & 97 & 48,7 \\
\hline Adolescencia media & 52 & 26,1 \\
Adolescencia tardía & 50 & 25,1 \\
Total & 199 & 100,0 \\
\hline
\end{tabular}

nica y 133 correspondían a población clínica, demostrando satisfactoria confiabilidad interna.

\section{Procedimiento}

La traducción del AAQ al idioma español siguió el procedimiento de traducción-retraducción, siendo evaluado por tres jueces expertos en la teoría del apego, quienes sugirieron modificaciones a nivel de la redacción de algunos ítems y respecto a las instrucciones, no existiendo indicaciones de eliminar ni agregar ítems. Esta primera versión del cuestionario fue aplicado a una muestra piloto, realizándose las últimas modificaciones al instrumento, especialmente asociadas a reemplazar en cada ítem "padre/madre" por un espacio en blanco “ ", lo que resultó más adecuados en cuanto, a solicitud de varios adolescentes, el apego primario no necesariamente está asociado al padre o la madre.

Finalmente se aplicó el cuestionario a un grupo de 215 adolescentes. Se eliminaron 16 cuestionarios pertenecientes a adolescentes de 5to año básico, por dificultad en la comprensión de ítems e instrucciones.

\section{Análisis estadístico}

Se evaluó, con el paquete estadístico SPSS v19, la consistencia interna del instrumento a través del Alpha de Cronbach, la validez de constructo con el procedimiento de análisis factorial y finalmente se elaboraron normas provisorias para su interpretación considerando diferencias de sexo y edad.

\section{Resultados}

\section{Análisis de confiabilidad}

El cuestionario en español demostró valores de consistencia interna similares, aun- 
que inferiores a la versión original. De acuerdo a los criterios propuestos por Guilford ${ }^{10}$, los valores de alfa de Cronbach para la escala Disponibilidad $(\alpha=0,74)$ resultan satisfactorios, mientras que para las escalas Cooperación en la Relación $(\alpha=0,64)$ y Señales de Enojo y Angustia $(\alpha=0,52)$ tendría valores que resultan medianamente satisfactorios (tabla 2).

\section{Análisis de la estructura factorial}

El análisis factorial, a través del método de extracción de componentes principales con rotación Varimax, para autovalores mayores a 1 , mostró la presencia de 3 factores, que explican el 61,71\% de la varianza total, y replican la estructura factorial del cuestionario original. Se utilizó este método, con el objetivo de determinar la cantidad de factores presentes en el cuestionario. La tabla 3 muestra los resultados obtenidos.

Incluyendo el mismo método de extracción se buscó identificar cómo se distribuyeron los ítems del cuestionario a través de tres factores principales, obteniéndose los siguientes resultados que se muestran en la siguiente matriz factorial en la tabla 4.

\section{Desarrollo de normas para la interpretación de resultados}

Se desarrollaron normas provisorias para la interpretación del AAQ identificando valores según sexo (masculino-femenino) y edad (adolescencia temprana, media y tardía).

Cómo se aprecia en la tabla 5, no se observan diferencias de sexo en ninguna de las escalas, no obstante, la tabla 6
Tabla 2. Análisis de confiabilidad

\begin{tabular}{|lcc|}
\hline & $\begin{array}{c}\alpha \text { estudio } \\
\text { original }\end{array}$ & $\begin{array}{c}\alpha \text { estudio de } \\
\text { adaptación }\end{array}$ \\
\hline $\begin{array}{l}\text { Escala Disponibilidad } \\
\text { Ítems 3-5-8 }\end{array}$ &, 80 &, 74 \\
$\begin{array}{l}\text { Escala Cooperación en la relación } \\
\text { Ítems 1-6-7 }\end{array}$ &, 74 &, 64 \\
$\begin{array}{l}\text { Escala Señales de Enojo y Angustia } \\
\text { Ítems 2-4-9 }\end{array}$ &, 62 &, 52 \\
\hline
\end{tabular}

Tabla 3. Porcentaje de Varianza explicada por factores

\begin{tabular}{|c|c|c|c|}
\hline \multirow[t]{2}{*}{ Componente } & \multicolumn{3}{|c|}{ Autovalores iniciales } \\
\hline & Total & $\%$ de varianza & $\%$ acumulado \\
\hline 1 & 3,086 & 34,287 & 34,287 \\
\hline 2 & 1,279 & 14,215 & 48,502 \\
\hline 3 & 1,189 & 13,208 & 61,710 \\
\hline 4 & 805 & 8,947 & 70,658 \\
\hline 5 & ,735 & 8,164 & 78,822 \\
\hline 6 & ,640 & 7,117 & 85,938 \\
\hline 7 &, 543 & 6,029 & 91,968 \\
\hline 8 & ,411 & 4,567 & 96,535 \\
\hline 9 & ,312 & 3,465 & 100,000 \\
\hline
\end{tabular}

Tabla 4. Matriz Factorial para AAQ versión en Español

\begin{tabular}{|ccccc|}
\hline & & \multicolumn{3}{c|}{ Componentes } \\
ítem & Escala & $\mathbf{1}$ & $\mathbf{2}$ & $\mathbf{3}$ \\
1 & $\mathrm{~B}$ &, 182 &, 785 &, 002 \\
2 & $\mathrm{C}$ &, 159 &,- 032 &, 649 \\
3 & $\mathrm{~A}$ &, 787 &, 139 &, 264 \\
4 & $\mathrm{C}$ &,- 136 &, 135 &, 818 \\
5 & $\mathrm{~A}$ &, 833 &, 126 &, 035 \\
6 & $\mathrm{~B}$ &, 206 &, 590 &, 206 \\
7 & $\mathrm{~B}$ &, 091 &, 852 &, 023 \\
8 & $\mathrm{~A}$ &, 714 &, 229 &,- 048 \\
9 & $\mathrm{C}$ &, 472 &, 213 &, 576 \\
\hline
\end{tabular}

$\mathrm{A}=$ disponibilidad; $\mathrm{B}=$ Cooperación; $\mathrm{C}=$ señales de enojo $\mathrm{y}$ angustia.
Tabla 5. Diferencias de sexo

\begin{tabular}{|lcc|}
\hline Escala & t & Sig (bilateral) \\
Disponibilidad & 1,881 &, 061 \\
Señales de Enojo y Angustia & 1,330 &, 185 \\
Cooperación & $-1,377$ &, 170 \\
\hline
\end{tabular}

$\mathrm{t}=\mathrm{t}$ de Student. ${ }^{*}=\mathrm{p}<0,05$.
Tabla 6. Diferencias de edad

\begin{tabular}{|lcc|}
\hline Escala & F & Sig \\
Disponibilidad &, 444 &, 642 \\
Señales de Enojo y Angustia &, 122 &, 885 \\
Cooperación & 4,05 &, $019^{*}$ \\
\hline
\end{tabular}

$\mathrm{F}=$ Análisis de Varianza. ${ }^{*}=\mathrm{p}<0,05$. 
Tabla 7. Normas provisorias para la Interpretación de Escalas del AAQ versión en Español

\begin{tabular}{|lcccc|}
\hline & Disponibilidad & Señales Enojo y Angustia & \multicolumn{2}{c|}{ Cooperación } \\
Promedio & 12,312 & 6,095 & $\mathbf{1 1}$ a 16 años & $\mathbf{1 7}$ a $\mathbf{2 0}$ años \\
Desviación estándar & 2,771 & 2,525 & 12,281 & 13,040 \\
Valores normales & 10 a 15 pts. & 0 a 8 pts. & 11 a 15 pts. & 12 a 15 pts. \\
\hline
\end{tabular}

muestra diferencias de edad para la escala "Cooperación", donde los adolescentes tardíos $(\mathrm{F}=4,05 ; \mathrm{p}=0,05)$ muestran valores más elevados para esta escala que los sujetos en adolescencia temprana y media.

La tabla 7 entrega normas provisorias para la interpretación de los resultados.

\section{Discusión}

Este estudio ha demostrado que el AAQ corresponde a un instrumento que mantiene características psicométricas similares al instrumento original al ser aplicado a una muestra de adolescentes de nuestro país.

Al comparar la versión traducida al español con el cuestionario original se observa que todos los ítems del primero, no obstante, tienen valores inferiores en cuanto a alfa de Cronbach. En el estudio actual la escala Disponibilidad muestra satisfactoria consistencia interna, mientras que para las escalas Cooperación y Señales de Enojo y Angustia este índice es medianamente satisfactorio.

Al considerar que esta última escala es la que obtiene valores más bajo en ambos estudios se sostiene que posiblemente requiera ser revisada. Su menor valor de consistencia interna podría tener relación con aspectos más bien teóricos en cuanto esta escala fue desarrollada a partir de la idea de Bowlby ${ }^{1}$ de que la ansiedad del infante producto de la no disponibilidad de una figura de apego cuando resulta necesaria, aumentaría la hostilidad hacia ésta ${ }^{11}$. Desde una mirada evolutiva, podríamos hipotetizar que estas observaciones no necesariamente podrían estar relacionadas al adolescente en cuanto, en palabras de Florenzano ${ }^{12}$, la cantidad y cualidad de las relaciones íntimas sufre cambios sustanciales durante esta etapa del desarrollo existiendo nuevas y diversas formas en el adolescente para hacer frente a la ansiedad.

Una segunda observación, tiene relación con la posible deseabilidad social que podría generar esta escala en algunos adolescentes, en cuanto hace explícita la posibilidad de conflicto real con padres, lo que sugeriría la necesidad de una inducción más detallada al cuestionario.

La escala Disponibilidad obtiene los mayores puntajes para ambos cuestionarios demostrando ser un factor que en ambas versiones resulta satisfactorio. No resulta extraña la alta confiabilidad de este factor en cuanto la literatura sobre apego ha puesto especial énfasis en la "disponibilidad" como uno de los aspectos centrales que definen este constructo.

En cuanto a la estructura factorial, se observa que la versión del cuestionario traducida al español igualmente posee 3 factores, lo que replica la estructura del cuestionario original. Así también, coincide la distribución de los ítems por escala, demostrándose la adecuación de esta versión del cuestionario.

La versión en inglés del AAQ muestra diferencias de sexo para las escalas Señales de Enojo y Angustia y Cooperación, sin embargo, en el actual estudio no se aprecian diferencias de sexo para ninguna escala. Un aparte de este estudio corresponde a la evaluación de diferencias de edad para las escalas del cuestionario, apreciándose diferencias para la escala Cooperación que no fueron consideradas en la versión en inglés. En este caso se observa que las medias de los adolescentes tardíos son significativamente superiores a los otros dos grupos etarios. Dado que esta escala evalúa la percepción del adolescente respecto a la coordinación que mantiene sobre las respuestas emocionales con sus padres ${ }^{4}$, es esperable que tal como se- 
ñala Allen $^{2}$ que al término de la adolescencia exista una mayor grado de regulación mutua entre los cuidadores y el adolescente. Resulta importante el hecho de que se haya indagado sobre diferencias etarias para esta versión del AAQ, en cuanto la literatura sobre la adolescencia ampliamente ha destacado el hecho de que la adolescencia no es un momento unitario del ciclo vital y posee diferencias que han llevado incluso a clasificar etapas distintas de la adolescencia.

El AAQ en su versión en español resulta un instrumento con características psicométricas similares a la versión original en inglés, con una estructura factorial idéntica y modificaciones a nivel de las instrucciones e ítems que a juicio de los investigadores son adecuadas para el grupo para el que está diseñado el instrumento. Pese a esto, es necesario recordar que la muestra con la que se realizó este estudio es significativamente menor a la muestra con que se elaboró el instrumento original, pudiendo existir sesgos en cuanto a la significación estadística de esta versión, quedando abierta la posibilidad de una estandarización en una muestra mayor y más representativa. Así también se debe señalar que la muestra que se utilizó para este estudio, por motivos de conveniencia en cuanto a su participación, no incorpora adolescentes pertenecientes a establecimientos municipalizados, o que estén fuera del sistema escolar. Finalmente, se señala que, en virtud de la falta de un instrumento que sirva como criterio al momento de realizar esta investigación, no fue posible evaluar la confiabilidad a través de una medida alternativa.

En conclusión, se espera que la versión en español del AAQ, resulte una herramienta eficaz para la investigación del apego en el plano de la adolescencia en virtud de la inexistencia, hasta la fecha, de instrumentos con estas ca- racterísticas en idioma español, y en especial, validados para nuestro país.

\section{Referencias}

1.- Bowlby J: La separación Afectiva. Buenos Aires, Editorial Paidós 1973.

2.- Allen JP, Land D: Attachment in adolescence. In J. Cassidy \& P. R. Shaver (Eds.), Handbook of attachment: theory, research, and clinical applications. New York: Guilford Press 1999.

3.- Feeney J, Noller P: Apego adulto. Bilbao: Descleé de Brouwer.

4.- West M, Rose S, Spreng S, Sheldon-Keller A, Adam $K$ : Adolescent attachment questionnaire: A brief assessment of attachment in adolescents. Journal of Youth and Adolescence 1998; 27: 661-73.

5.- Brown L, Wright J: The relationship between attachment strategies and psychopathology in adolescence. Journal of Psychology and Psychotherapy 2003; 76 (4): 351-67.

6.- Pardo M, Pineda S, Carrillo S, Castro J: Análisis Psicométrico del Inventario de Apego con Padres y Pares en una Muestra de Adolescentes Colombianos. Revista Interamericana de Psicología 2006; 40 (3): 289-302.

7.- Martínez C, Santelices M: Evaluación del Apego en el Adulto: Una Revisión. Revista Psyche, Pontificia Universidad Católica de Chile 2005; 14: 181-91.

8.- George C, Kaplan M, Main M: Adult attachment interview. Unpublished manuscript, University of California, Berkeley, 1985.

9.- Ainsworth M, Blehar M, Waters E, Walls S: Patterns of attachment: A psychological study of the strange situation. Hillsdale, NJ: Erlbaum, 1978.

10.- Guilford J: Psychometric methods. New York: Mc Graw Hill 1973.

11.- Lecannelier F: Apego e Intersubjetividad. Segunda Parte: La Teoría del Apego. Ediciones LOM (2009).

12.- Florenzano R: El adolescente y sus conductas de riesgo. Santiago, Chile: Editorial Pontificia Universidad Católica de Chile, 2005. 